\title{
Ciências da Saúde
}

\section{A IMPORTÂNCIA DA ATENÇÃO FARMACÊUTICA AO IDOSO}

\author{
THE IMPORTANCE OF PHARMACEUTICAL IN ASSISTANCE TO THE ELDERLY IN \\ DISPENSATION
}

\begin{abstract}
Allan Gabriel Moura1; LorenaGabriel Moura²; Vera Lúcia Matias Geron³; André Tomaz Terra Junior ${ }^{4}$; Regiane Rossi Oliveira Lima ${ }^{5}$.
\end{abstract}

RESUMO: O trabalho se apresenta com a temática relacionada a importância do farmacêutico na assistência ao idoso na dispensação. O objetivo do presente trabalho é abordar de maneira enfática e precisa acerca do processo de envelhecimento, abordando um pequeno histórico da pessoa idosa, bem como fragilidades, o conceito de dispensação medicamentosa, e a importância do farmacêutico nesta seara, haja vista que o mesmo possui a obrigação de acompanhar e fornecer atenção farmacêutica e dispor a medicação para a comunidade. Quando se tratando do idoso é muito mais importante, pois os mesmos possuem suas dificuldades e limitações que podem acarretar diversos problemas no ato da medicação, na leitura do medicamento, na bula dentre outros fatores que devem ser considerados pelo profissional farmacêutico ao fazer este atendimento ao idoso. $O$ idoso necessita de uma atenção especial e cautelosa, haja vista que os mesmos geralmente se medicam por conta própria, devendo o profissional farmacêutico estar preparado também para lidar com questões que vão além de sua ciência, como bom atendimento, respeito e paciência com o atendimento.

Palavras-Chaves: Atenção Farmacêutica, Paciente Idoso; Assistência Farmacêutica, Pacientes Especiais.

\footnotetext{
ABSTRACT: The present paper deals with the importance of the pharmacist's assistance to the elderly in dispensing. The aim of this work is to emphatically address the aging of the elderly, as well as their fragilities, the concept of dispensing medication, and the importance of the pharmacist in this area, considering that it has the duty to monitor and provide pharmaceutical assistance and medication to the community. When it comes to the elderly, it is much more important, since they have their difficulties and limitations that can lead to several problems in the medication, reading the medication, the package insert among other

${ }^{1}$ Acadêmico do curso de Farmácia da Faculdade de Educação e Meio Ambiente - FAEMA- Ariquemes - RO;

${ }^{2}$ Acadêmico do curso de Farmácia da Faculdade de Educação e Meio Ambiente - FAEMA- Ariquemes - RO;

${ }^{3}$ Mestre em Biologia dos Agentes Infecciosos e Parasitários pela Universidade Federal do Pará -UFPA. Docente e Coordenadora do curso de graduação em Farmácia da FAEMA - RO;

${ }^{4}$ Mestre em Oncologia Clínica, Células-tronco e Terapia Celular pela Faculdade de Medicina de Ribeirão Preto - USP. Docente do Curso de Farmácia na Faculdade de Educação e Meio Ambiente - FAEMA, Ariquemes $\mathrm{RO}$;

${ }^{5}$ Especialista em Farmácia Clinica. Docente e Coordenadora dos Laboratórios Didáticos da FAEMA-RO.
} 
factors that should be considered by the pharmacist when doing this care old man. The elderly need special and cautious attention, since they usually medicate themselves, and the pharmacist should also be prepared to deal with issues that go beyond their science, such as good care, respect and patience with the attendance.

Words-Keys: Pharmacist; Assistance; Elderly, Dispensing.

\section{APRESENTAÇÃO}

O conceito de idoso está formado de duas maneiras, para nações em desenvolvimento o mesmo é conceituado como sendo, pessoas com idade de sessenta anos acima. $E$ em países desenvolvidos são pessoas idosas aquelas com idade superior a 65 anos. (1)

Os idosos apresentam dificuldade e limitações, haja vista que os mesmos vão se desgastando ao longo de suas vidas, parte da musculatura tem comprometimento, a visão possui fragilidades, bem como apresentam dificuldades na leitura e seu corpo já não reage como antes. (2)

A dispensação e o atendimento são muito importantes na vida dos provectos, e faz parte de um processo de atenção ao ancião, onde esse processo deve ser entendido como um todo, ou seja, desde a medicação até o atendimento ao pessoa com idade avançada, que é o que vai fazer a diferença para o mesmo no momento de utilizar a medicação, o farmacêutico deve estar preparado para enfrentar o processo de atendimento com muito cuidado e paciência, pois a população idosa que depende do uso de medicamentos possuem dificuldades as vezes para compreender sobre a forma correta de utilização do medicamento(3).

O objetivo do presente trabalho é abordar de maneira enfática e precisa acerca do processo de envelhecimento, abordando um pequeno histórico da pessoa idosa, bem como fragilidades, 0 conceito de dispensação medicamentosa, e a importância do farmacêutico nesta seara, haja vista que o mesmo possui a obrigação de acompanhar e fornecer atenção farmacêutica e dispor a medicação para a comunidade.

O presente trabalho se encontra pautado em uma temática muito importante que é importância do farmacêutico na dispensação medicamentosa para os idosos e sua contribuição para melhoria na terapêutica bem como adesão e utilização.

\section{METODOLOGIA}


O trabalho exposto foi realizado com uma pesquisa bibliográfica onde foi possível verificar os ensinamentos de autores que por vezes já vinham discutindo acerca do tema abordado, dentre outros que esboçam sua preocupação com a dispensação de medicamentos para os idosos e acredita ser relevante para a discussão pesquisas com o intuito de auxiliar outros trabalhos e vislumbrar mais informações acerca do tema.

A busca do material bibliográfico deu-se nos seguintes documentos aplicado através da busca de materiais de dados eletrônicos, a saber: Google Acadêmico (pesquisa avançada) e Scientific Eletronic Library Online SciELO, e livros, que relata os assuntos em questão, os quais contribuíram a elevar a melhor compreensão.

\section{REVISÃO DE LITERATURA}

\subsection{Definição de Atenção Farmacêutica} (AF)

Dispensação de medicamentos é o ato onde $o$ farmacêutico tem a habilidade de entregar ao paciente algum medicamento, podendo ser um ou mais, visto que esse procedimento segue suas regras e deve ser observado com cautela pelo profissional. Com a evolução dessa ciência outras nomenclaturas foram sendo adotadas e, que dizem a dispensação como sendo a "Atenção Farmacêutica", a atenção farmacêutica não se baseia somente no ato de passar o medicamento, mas todo o processo em que o paciente deve ingerir o medicamento bem como o ato de orientar o idoso, auxilia-lo de todas as maneiras possíveis em sua medicação, (4).

O farmacêutico é o profissional responsável por atender as pessoas que necessitam de medicamentos, podendo estes ser ou não prescritos por médicos, dentistas etc., porém há casos em que somente podem prescrever ao paciente os medicamentos que estão sob controle do órgão competente. Entretanto, na maioria dos casos os medicamentos são de controle especial e necessitam de atenção prioritária do farmacêutico(5).

\subsection{Envelhecimento}

O processo da vida é dividido em várias etapas até chegar à fase idosa, momento este que a debilidade é o fator que acarreta diversos problemas de saúde tanto mentais e físicos. Até mesmo no nascimento 0 ser humano passa por diversos momentos, porém os idosos apresentam mais dificuldades relativas à sua saúde(6). 
A população brasileira idosa vem aumentando gradativamente a cada ano que se passa haja vista que há alguns anos o país era composto mais por pessoas jovens e crianças, porém percebe-se ainda que cada dia mais os pais tendem a ter menos filhos, por diversos motivos, como psicológicos, pessoais, e financeiros. O Brasil vem apresentando um crescente percentual de idoso, isso faz com que seja necessária uma atenção especial para esta classe que vem aumentando a cada dia(6).

O envelhecimento vem ocorrendo de maneira precoce, onde as pessoas se tornam idosas muito mais cedo do que ocorria em tempos anteriores, vários são os fatores que asseguram essa ocorrência, visto que alguns deles derivam de falta de atividades físicas, como preocupação no cotidiano, vida corrida, estresse emocional e físico(7).

Este momento vivido pelos idosos é tido por vezes como uma fase de derrota, visto que os mesmos não possuem a mesma força, aptidão, mesmo porte físico e etc, (12).

\subsection{Fragilidades do idoso $\mathrm{X}$ atendimento} farmacêutico

A fragilidade no idoso acontece por motivos realmente que ocorrem com 0 tempo como o cansaço físico e mental, falta de coordenação motora, falta de atividade física no cotidiano(8).

É nessa fase que o idoso mais busca ajuda nas farmácias visto que a consulta médica não ocorre com tanta frequência e que os mesmos nem sempre podem estar buscando auxílio médico com acuidade, assim priorizam a busca por tratamentos alternativos direto nos estabelecimentos farmacêuticos, isso pode gerar danos a própria saúde, sendo que o mesmo com suas debilidades nem sempre consegue acompanhar $o$ modo de manuseio do medicamento, o medicamento ao mesmo tempo em que é benéfico pode se tornar uma ferramenta perigosa para os seres humanos(9).

Nesta seara os farmacêuticos devem atender o a pessoa idosa de maneira especial, visando sempre seu bem estar social e com cuidado no uso de medicamentos. Em especial para os idosos, a recomendação acerca do uso racional de medicamentos, é pratica importante em virtude de múltiplas patologias, necessitando buscar diversos tratamentos devido as doenças que os apanham durante a vida fazendo com que isso leve os mesmos a se medicarem com alguma frequência(10). 
$O$ atendimento ao idoso deve ser completo e sempre ativo, o farmacêutico deve sempre compreender todo o conjunto de necessidade para com seu cliente idoso, percebendo que o mesmo necessita de uma atenção dobrada quando ao uso de medicamentos, perpassando pelo ponto de vista medicamentoso, o mesmo deve se sobressair, entrevistando o paciente para que não reste dúvidas de qual medicamento o mesmo deve ingerir(10).

Os farmacêuticos têm que se habituar a ter uma percepção mais aguçada em relação a alergias, e perguntar se o paciente já ingeriu outros medicamentos, como tomava, aplicando todas as informações no momento da dispensação do medicamento para o mesmo.

Em meio a dispensação do medicamento se inclui a dosagem do medicamento para o ancião, devendo este profissional ater para os cuidados básicos com este idoso que nem sempre vai ingerir um medicamento com a mesma dosagem de uma adulto jovem, percebe-se também que neste contexto o farmacêutico deve verificar e anotar na caixa do medicamento com letra legível a maneira e o os horários em que 0 idoso irá ingerir a medicação(11).
$\mathrm{Na}$ dispensação devem ser levados em consideração os horários em que o idoso deve tomar seus medicamentos, cabendo ao farmacêutico com muita cautela e respeito, informar além da prescrição na embalagem do medicamento, orientar o idoso nesta etapa da dispensação medicamentosa. Nesta fase compreende-se que os idosos já possuem uma visão prejudicada pelo tempo de vivência e de problemas de saúde que muitas vezes os prejudicam no momento da ingestão dos medicamentos principalmente na concentração a ser ingerida(12).

A dispensação dos medicamentos faz parte do atendimento farmacêutico, alguns autores têm as mesmas atividades interligadas uma com a outra. É nesse momento que o farmacêutico deverá fazer uma análise contextual com 0 idoso alertando o mesmo de possíveis reações, forma ideal de ingerir o medicamento, dicas para não se esquecer de tomar o medicamento, como deve ser ingerido em que período dentre outras informações necessárias(11).

\subsection{Estratégias farmacêuticas na dispensação de medicamentos}

O profissional em farmácia ao lidar com o público, principalmente com grupos 
de idosos, deve usar ferramentas que podem ser trabalhadas para auxiliar 0 idoso no uso de sua medicação, dever esse definido como atenção farmacêutica essa atenção que o profissional em seu atendimento faz com o idoso podem gerar diversos benefícios para o mesmo(8).

As estratégias devem estar atreladas ao bom atendimento, ou seja, maneiras de chamar a atenção do idoso para alguns pontos importantes ao tomar sua medicação, essas metodologias devem estar atreladas a boa formação do profissional que trabalha para que 0 paciente idoso se medique da melhor maneira possível evitando diversos problemas de saúde futuros. Por vezes o Ministério da saúde adverte aos farmacêuticos que os mesmos devem usar estratégias, maneiras de conduzir o idoso para melhor receber sua medicação, algumas dessas estratégias é o acompanhamento individual do idoso, fazendo com que a explicação do conteúdo seja mais completa e clara possível(13).

As estratégias realizadas pelos profissionais em saúde (farmacêuticos) devem estar ligada diretamente com o que preconiza a medicação estipulada pelo médico que é quem diagnosticou, o tratamento é uma fase importante na recuperação de um idoso que busca medicação, porém deve ser acompanhada e mediada(13).

Os farmacêuticos podem ainda estarem aplicando metodologias como tabelas e horários, onde ficara mais fácil para que $o$ idoso compreenda a maneira e tempo correto dos seus medicamentos. $\mathrm{O}$ trabalho realizado em equipe é uma estratégia consoante que visa sempre o bom atendimento, visto que se possível o farmacêutico obtiver as informações dos pacientes, o mesmo poderá acompanhar mais de perto a jornada do profissional, visto que 0 tratamento que o mesmo estiver realizando terá mais eficácia(14).

As estratégias utilizadas na dispensação do medicamento a idosos vão além a informação contida do âmbito de trabalho, pois nem sempre o idoso possui uma família que o apoia e auxilia em sua medicação, fazendo com que muitas vezes o idoso não consegue acompanhar o tratamento que deve ser feito, desta maneira o farmacêutico irá atuar na verificação de como vive esse idoso, para ser mais cauteloso na dispensação do medicamento(15).

Outras possíveis como não ofertar número elevado de medicamentos, conhecer o paciente e se preocupar com o 
bem estar físico e psicológico do mesmo para que então consiga de maneira satisfatória atingir o objetivo de atuar na dispensação de maneira completa. Há casos em que o idoso é cuidado por netos ou sobrinhos que nem sempre esses possuem entendimento suficiente para medicar o idoso fazendo com que a medicação não seja aplicada de maneira correta(15).

Para que o farmacêutico possa ser mais efetivo na dispensação é fundamental que o mesmo conheça o histórico dos idosos, adotando essa metodologia, os tratamentos realizados pelos idosos podem se tornar mais eficazes para os mesmos(16).

Um dos problemas que afetam 0 tratamento de idosos é a falta dos familiares competentes nesse acompanhamento, fazendo com que os idosos se sintam excluídos e não finalizam seus tratamentos, fazendo com que em alguns casos as doenças se propaguem ainda mais(17).

O farmacêutico é o responsável pela dispensação, acompanhamento, atenção e principalmente o responsável pela medicação oferecida para os pacientes(18).
Percebe-se que além dos jovens os idosos também se medicam inclusive usando sedativos, bem como percebe-se que esses medicamentos só podem ser prescritos por profissional (18).

O uso de medicamento de maneira irracional pelo idoso pode causar seu óbito ou deixar sequelas, por esse fator também é interessante a presença do farmacêutico nesse importante processo. (19)

\section{CONSIDERAÇÕES FINAIS}

Após a revisão de literatura, percebeu-se que o farmacêutico como atuante frente as farmácias brasileiras, por estarem ligados diretamente com a sociedade principalmente idosa que consome uma grande quantidade de medicamentos, precisa estar mais informado quanto a importância desse atendimento ao idoso, e que este depende do mesmo não somente como um profissional, mas como alguém que tenha competência e capacidade de atende-lo com respeito, paciência e muita cautela, respeitando os limites que os mesmos possuem.

Ainda neste contexto é possível verificar que os profissionais farmacêuticos possuem importância para a medicação farmacológica dos idosos e assim deveriam se preparar de maneira mais 
ampla e humanitária neste atendimento, não deixando soar a negatividade de seu profissionalismo.

\section{REFERÊNCIAS}

1. Santos Silvana. Sidiney. Costa. Concepções teórico Concepções teóricofilosóficas sobre -filosóficas sobre envelhecimento, velhice, idoso e enfermagem envelhecimento, velhice, idoso e enfermagem gerontogeriátrica ontogeriátrica (2010). Disponível em: http://www.scielo.br/pdf/reben/v63n6/25.pd f. Acesso em: 29/03/2017.

2. Nardi, Edileuza, Fátima, Rosina et al . Associação Entre A Incapacidade Funcional Do Idoso E A Sobrecarga do Cuidador familiar. Disponível em: http://www .scielo. br/ pdf/rlae/v21n5 /pt_0104-1169 -rlae-21-05- 1096.pdf. Acesso em:28/03/2017.

3. Galato, Dayan et al. A dispensação de medicamentos: uma reflexão sobre 0 processo para prevenção, identificação e resolução de problemas relacionados à farmacoterapia.(2008). Revista scielo. Disponível em: http://www.scielo.br/pdf/rbcf/v44n3/a 17v44n3.pdf. Acesso em: 21/03/2017.

4. Hepler, et al.Oportunidades e responsabilidades na assistência farmacêutica. Revista Am. J. Hosp Pharm, (1990). Disponível em: http://www.scielo.br/scielo.php?script=sci arttext\&pid= S1516-93322008000400006. Acesso em: 18/03/2017.

5. Angonesi, Daniela Marcela; Rennó Unes Pereira. (2011). Dispensação Farmacêutica: proposta de um modelo para a prática. Disponível em: http://www.scielo.br/pdf/csc/v16n9/ a24v16n9. pdf. Acesso em: 20/03/2017.

6. Kuznier, Tatiane, Preter. O significado do envelhecimento e do cuidado para o idoso hospitalizado e as possibilidades do cuidado de si. (2007). 124 f. Dissertação (Mestrado) - Curso de Programa de Pósgraduação em Enfermagem.

7. Papaléo, Netto. Gerontologia. São Paulo: Atheneu, 1996.

8. Queiroz, Zally. Cuidando do idoso: uma abordagem social. O Mundo da Saúde, São Paulo, 2000.

9. Le Sage .Polipharmacy in geriatric patients. Nursing Clinics of North America, Philadelphia, v.26, p.273-290, 1991.

10. Tapia-Conyer et al. Consumo de drogas médicas en poblacion de 60 a 65 años en México. Salud Pública de México, México, v.38, n.6, 1996.

11. Assis, Aldina Julieta Cruz de. A Importância do farmacêutico comunitário na dispensação de medicamentos entre idosos na rede pública de saúde: Revisão de Literatura. (2014). Revista Especialize On-line IPOG - Goiânia - 8를 Edição no 009 Vol.01/2014 dezembro/2014. Disponível em: file:///C: /Users/Usu\%C3\% A1 rio /Downloads /a -importancia-do farmaceutico- comunitario- nadispensacao- de-medicamentos-entreidosos-na-rede- publica-de-saude-revisaode-literatura-1081511.pdf. Acesso em: 20/03/2017.

12. Andrade Marcieni Ataide de; Silva Marcos Valério Santos da; Freitas Osvaldo 
de. (2016). Assistência Farmacêutica como Estratégia para o Uso Racional de Medicamentos em Idosos. Disponível em: http://www.uel.br/proppg / /portal/ pages/arquivos/

pesquisa/semina/pdf/semina_25_1_20_17. pdf . Acesso em 10/04/2017.

13. Brasil. Ministério da Saúde. Secretaria de Atenção à Saúde. Departamento de Atenção Básica. Saúde. Diretrizes do NASF: núcleo de apoio à saúde da família. Brasília, DF, 2009. Disponível em: fttp://bvsms.saude.gov.br/ bvs/publicações /caderno_atencao_basica_diretrizes nasf.pd. Acesso em:10/04/2017.

14. Panstein, R.; Weber, C. A. T. Avaliação de satisfação da gestão da Assistência Farmacêutica Básica em Jaraguá do sul no ano de 2007. Revista de Saúde Pública de Santa Catarina, Florianópolis, v. 3, n. 2, p. 46-62, 2010. Disponível em: Acesso em: 23/03/2017.

15. Centa, et al. Programa de saúde da família sob 0 olhar da equipe multidisciplinar. (2003). Família, Saúde e Desenvolvimento, Curitiba, v. 5,n. 2., p. 103-113.
16. Athayde, et al. Possibilidades do uso do programa no trabalho cotidiano dos médicos das equipes de saúde da família de Londrina. Revista Espaço para a Saúde, Londrina, v.6, n. 2, 2005.

17. Cipolle, et al. ejercicio de la atención farmacêutica. Madrid: McgrawRio, 2000.

18. Marin, et al. Assistência Farmacêutica para gerentes municipais. Brasília: Organização Pan-Americana da Saúde/Organização Mundial da Saúde, 2003.

19. Santos, Valdeci. O que é e como fazer "revisão da literatura" na pesquisa teológica. Fides reformata xvii, n. 1. 2012.

18. Opaleye, Elias. et al. Nonprescribed use of tranquilizers or sedatives by adolescents: a Brazilian national survey. BMC public health, London, v.13, 2013.

19. Fernandes Wendel Simões; Cembranelli, Julio César. Automedicação e o uso irracional de medicamentos: o papel do Profissional farmacêutico no combate a essas práticas.2015. Disponível em: 